\title{
Harmonic Analysis of Power System Based on Nuttall self-convolution Window Triple-spectral-line Interpolation FFT
}

\author{
Harmonic analysis of power system
}

\author{
Yifan Zhu' ${ }^{1}$, Yue Wang, ${ }^{2, *}$ Ting Lin $^{2}$, Chengcheng Feng ${ }^{2}$, Jiong Chen ${ }^{2}$ and Yuan Gao ${ }^{2}$ \\ ${ }^{1}$ Shanghai Power Grid Electric Power Research Institute, Shanghai 200000, China \\ ${ }^{2}$ School of Electrical Engineering, Shanghai University of Electric Power, Shanghai 200082, China \\ ${ }^{*}$ Corresponding author
}

\begin{abstract}
Utilization of window functions and interpolation algorithms for Fast Fourier transform can effectively restrain the spectral leakage and picket fence effect in situation of nonsynchronized sampling and non-interger cycle truncation. In this paper, an improved FFT approach for harmonic based on Nuttall self-convolution window triple-spectral-line interpolation is improved. The simulation results showed that the proposed method have a higher accuracy of harmonic analysis and could improve the anti-noise performance. In the paper, triplespectral-line interpolation correction algorithm for Nuttall selfconvolution window is used to improve the accuracy and anti noise is the latest research results, by the simulation can prove the superiority of this algorithm.
\end{abstract}

Keywords- Nuttall self-convolution window; triple-spectral-line interpolation; harmonic; spectral leakage; non-synchronized sampling

\section{INTRODUCTION}

With the wide application of various nonlinear loads, especially the power electronic equipments in power system, harmonic pollution is becoming more and more serious ${ }^{[1]}$. Fast Fourier transform (FFT) algorithm ${ }^{[1,2]}$ can make the number of multiplications needed to compute greatly reduced, especially when the sampling points are huge, the FFT algorithm of calculation speed is more faster. Utilization of window functions and interpolation algorithms for Fast Fourier transform can effectively restrain the spectral leakage and picket fence effect in situation of non-synchronized sampling and non-interger cycle truncation, the common window function including Rife-Vincent windows ${ }^{[3-4]}$, Nuttall windows $^{[5]}$, Nuttall self-convolution window $^{[2,6,7]}$. the appropriate window function can improve the restrain the spectral leakage and picket fence effect, but due to the complex signal and the window function character restrictions, commonly used interpolation algorithm to improve accuracy, including double-spectral-line interpolateon ${ }^{[1]}$ and triplespectral-line interpolation ${ }^{[8-9]}$ algorithm.

\section{HARMONIC RESEARCH METHOD}

A. Nuttall self-convolution window and behavior of sidelobe

The spectral characteristics of the window include the main lobe width, the side lobe peak level, and the side lobe attenuation rate. The main lobe width is wider, and the frequency resolution is lower. The side lobe is bigger, the frequency will leakage more. And the attenuation of the side lobe is faster, the ability of window to strain leakage is better. Therefore, choosing the 4 order 5 function Nuttall window as the parent window, then set up Nuttall self-convolution window triple-spectral-line interpolation.

The time domain of the Nuttall window is represented as

$$
w_{N}(n)=\sum_{m=0}^{M-1}(-1)^{m} b_{m} \cos \left(\frac{2 \pi}{N} m n\right)
$$

In the formula, $M$ is the length of the window function; $b_{0}=0.3125, b_{1}=0.46875, b_{2}=0.1875, b_{3}=0.03125$; $n=0,1, \ldots, N^{\prime}-1$; The constraints for $b_{m}$ is

$$
\left\{\begin{array}{l}
\sum_{m=0}^{M-1} b_{m}=1 \\
\sum_{m=0}^{M-1}(-1)^{m} b_{m}=0
\end{array}\right.
$$

The spectral function of the Nuttall window is

$w_{N}(\omega)=\sum_{m=0}^{M-1}(-1)^{m} b_{m}\left[w_{R}\left(\omega-\frac{2 \pi}{N} m\right)+w_{R}\left(\omega+\frac{2 \pi}{N} m\right)\right](3)$

In the formula, $W_{R}(\omega)$ is the frequency spectrum function of the rectangular window, the expression is 


$$
W_{R}(\omega)=\frac{\sin \left(\omega N^{\prime} / 2\right)}{\sin (\omega / 2)} e^{-j \omega\left(N^{\prime}-1\right) / 2}
$$

The Nuttall self-convolution window defines several identical Nuttall windows for time-domain convolution operations, the formula is

$$
w_{C}(n)=\underbrace{w_{N}(n) * w_{N}(n) * \ldots * w_{N}(n)}_{p}
$$

In the formula, $p$ is the number of Nuttall windows involved in convolution, which is called the order of Nuttall selfconvolution window. Before operation, the length of Nuttall is $N$,through the convolution operation the length of the Nuttall self-convolution window has become $p-1$, so, it is necessary to make zero padding before and after convolution sequences.

In the frequency domain operation, the Nuttall selfconvolution window is defined as the power of the spectral function of the Nuttall window, the formula is

$$
\begin{aligned}
& W_{N}^{p}(\omega)=\left\{\sum _ { m = 0 } ^ { M - 1 } ( - 1 ) ^ { m } \frac { b _ { m } } { 2 } \left[e^{-j \frac{\omega_{\omega-m}\left(N^{\prime}-1\right)}{2}} \frac{\sin \left(\omega_{\omega-m} N / 2\right)}{\sin \left(\omega_{\omega-m} / 2\right)}+\right.\right. \\
& \left.\left.e^{-j \frac{\omega_{\omega+m}\left(N^{\prime}-1\right)}{2}} \frac{\sin \left(\omega_{\omega+m} N / 2\right)}{\sin \left(\omega_{\omega+m} / 2\right)}\right]\right\}^{p}
\end{aligned}
$$

Take $p=2$ for example, when $N=50$, Figure 1 is the frequency response of the Nuttall self-convolution windows. In figure 1 , the Side lobe level peak is $-121.9 \mathrm{~dB}$, the Side lobe attenuation rate is $84 \mathrm{~dB} /$ oct. The Side lobe attenuation rate of Nuttall self-convolution windows is better than Nuttall window and Rife-Vincent window.

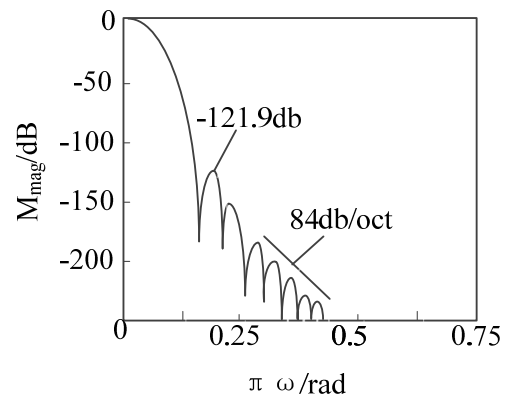

FIGURE I FREQUENCY RESPONSE OF THE NUTTALL SELFCONVOLUTION WINDOWS

\section{B. Nuttall self-convolution window triple-spectral-line interpolation FFT}

In power system , enter a time-domain harmonic signal $x(t)$ is

$$
x(t)=\sum_{h=1}^{H} A_{h} \sin \left(2 \pi r_{h} f_{1} t+\varphi_{h}\right)
$$

In the formula, $H$ is the number of harmonic. When $h=1, f_{1}, A_{1}, \varphi_{1}$ are the fundamental wave of frequency $(\mathrm{Hz})$, amplitude(V) and initial phase $\left({ }^{\circ}\right)$. When $h \neq 1, r_{h}$ represents the number of harmonics in item $h . A_{h}, \varphi_{h}$ represent item $h$ harmonic amplitude (V) and phase $\left({ }^{\circ}\right)$.

The signal $x(t)$ is sampled at a sampling frequency of $f_{s}$ to obtain discrete time signals, which is

$$
x(n)=\sum_{h=1}^{H} A_{h} \sin \left(\frac{2 \pi r_{h} f_{1} n}{f_{s}}+\varphi_{h}\right)
$$

For signal $x(n)$, collecting several periodic signals by Nuttall self-convolution window, then we can obtain

$$
x_{w}(n)=x(n) w_{N}^{P}(n)
$$

In the formula, $w_{N}^{P}(n)$ is the Nuttall self-convolution window in frequency domain.

For $x_{w}(n)$, do it with discrete fast Fourier transform (DTFT), we can obtain

$$
\begin{aligned}
& x_{w}(f)=\sum_{-\infty}^{+\infty} x(n) w_{N}^{p}(n) e^{-j 2 \pi n f} \\
& =\sum_{h=1}^{H} \frac{A_{h}}{2 j}\left[e^{j \varphi_{h}} W_{R}^{p}\left(2 \pi\left(f-r_{h} f_{1}\right) / f_{s}\right)-\right. \\
& \left.e^{-j \varphi_{h}} W_{R}^{P}\left(2 \pi\left(f+r_{h} f_{1}\right) / f_{s}\right)\right]
\end{aligned}
$$

In the actual measurement of power system harmonics, in order to obtain the harmonic of a certain item, only one point is considered and the influence of the other points is neglected, therefore, the formula for $y_{w}$ is

$$
y_{w}(k \Delta f)=\frac{A_{i}}{2 j}\left[e^{j \varphi_{i}} W_{R}^{p}\left(2 \pi\left(k-k_{i}\right) / N\right)\right]
$$

In situation of non-synchronized sampling, the signals could produce spectral leakage and picket fence effect. In this time, Sampling point $f_{i}=k_{i} \Delta f$ barely fall on the peak frequency line point. Suppose that the maximum spectral line sampled near the peak frequency point is $k_{i 1}$, at the same time, the spectrum of the left and right sides $k_{i 0}$ and $k_{i 2}$ also contains rich spectral information, its size is related to $k_{i 0}\left(=k_{i 1}-1\right) \leq k_{i 1} \leq k_{i 2}\left(=k_{i 1}+1\right)$. The amplitudes of the 3 lines are recorded as $y_{0}=\left|y_{w}\left(k_{i 0} \Delta f\right)\right|, y_{1}=\left|y_{w}\left(k_{i 1} \Delta f\right)\right|$ and 
$y_{2}=\left|y_{w}\left(k_{i 2} \Delta f\right)\right|$

Because of $0 \leq k_{i}-k_{i 1} \leq 1$, for convenience of calculation, introducing two variables $\alpha$ and $\beta$.To make that $\alpha=k_{i}-k_{i 1}-0.5(\alpha \in[-0.5,0.5]) \quad, \quad \beta=\left(y_{2}-y_{0}\right) / y_{1} \quad$,By formula (11),we can obtain

$$
\begin{aligned}
\beta & =\frac{y_{2}-y_{0}}{y_{1}} \\
& =\frac{\left|W_{N}^{p}(2 \pi(-\alpha+0.5) / N)\right|-\left|W_{N}^{P}(2 \pi(-\alpha-1.5) / N)\right|}{\left|W_{N}^{p}(2 \pi(-\alpha-0.5) / N)\right|}
\end{aligned}
$$

At this point, $\alpha$ is the independent variable, $\beta$ is the dependent variable, and the inverse function is $\alpha=f^{-1}(\beta)$. Take a set of $\alpha$ values within $\alpha \in[-0.5,0.5]$, getting the corresponding $\beta$,then calling the function $\operatorname{polyfit}(\beta, \alpha, m)$ to do polynomial inverse fitting, it can approximation formula of the function $\alpha=F(\beta)$.

The interpolation correction formula for item i frequency is

$$
f_{i}=k_{i} \Delta f=\left(\alpha+k_{i 1}+0.5\right) \Delta f
$$

In order to obtain the minimum measurement error, the amplitude frequency is interpolated by windowed interpolation, the maximum spectral line $k_{i 1}$ contain a wealth of information, but the left and right sides $k_{i 0}$ and $k_{i 2}$ still contain lots of information. In order to minimize the error, the triple-spectralline interpolation is used to correct the formula.

Weighted average calculation of three spectral lines, the amplitude correction formula is obtained

$$
A_{i}=N^{-p}\left(y_{2}+2 y_{1}+y_{0}\right) g(\alpha)
$$

The correction formula for harmonic phase is

$$
\varphi_{i}=\arg \left[y_{w}\left(k_{i h} \Delta f\right)\right]+\frac{\pi}{2}-\arg \left[W_{R}^{p}(-\alpha-0.5)\right]
$$

In the formula, $h=0,1,2$.

In the time $p=2$, based on the 4 order 5 order the Nuttall self-convolution window triple-spectral-line interpolation is

$$
\begin{aligned}
& \alpha=2.017780726618934 \beta-0.14416912519588 \beta^{3}+ \\
& 0.020680914246738 \beta^{5}-0.003614378944554 \beta^{7}
\end{aligned}
$$

$$
\begin{aligned}
& g(\alpha)=21.93553832925077+2.726188198628158 \alpha^{2}+ \\
& 0.176288053666237 \alpha^{4}+0.008047452065558 \alpha^{6}
\end{aligned}
$$

\section{SimUlation RESUlts ANALYSIS}

\section{A. Harmonic FFT Analysis}

Using simulation signals as

$$
x(n)=\sum_{h=1}^{H} A_{h} \sin \left(2 \pi r_{h} f_{1} n / f_{s}+\varphi_{h}\right)
$$

The sampling frequency is $1500 \mathrm{~Hz}$, the fundamental frequency is $50.1 \mathrm{~Hz}$, and the sampling number $\mathrm{N}$ is 1024 . Specific data information is shown in table 1 .

TABLE I. FREQUENCY, AMPLITUDE AND PHASE OF THE FUNDAMENTAL WAVE AND EVERY HARMONIC SIGNAL

\begin{tabular}{cccc}
\hline Harmonic & Frequency/Hz & amplitude/V & phase/( $\left.{ }^{\circ}\right)$ \\
\hline 1 & 50.1 & 220 & 10 \\
3 & 150.3 & 35 & 20 \\
5 & 250.5 & 27 & 40 \\
7 & 350.7 & 24 & 60 \\
9 & 450.9 & 20 & 90 \\
11 & 551.1 & 17 & 130 \\
\hline
\end{tabular}

To obtain the accurate harmonic signal, based on the Nuttall self-convolution window triple-spectral-line interpolation, getting the truncated signal. table 2 is the date of it .

TABLE II. AMPLITUDE ERRORS OF DIFFERENT WINDOW INTERPOLATION ALGORITHMS

\begin{tabular}{cccc}
\hline Harmonic & Blackman & Nuttall & Nuttall self-convolution window \\
\hline 1 & $2.9 \mathrm{e}-7$ & $-1.09 \mathrm{e}-7$ & $1.04 \mathrm{e}-08$ \\
3 & $3.8 \mathrm{e}-7$ & $6.40 \mathrm{e}-8$ & $8.57 \mathrm{e}-09$ \\
5 & $9.0 \mathrm{e}-8$ & $-1.30 \mathrm{e}-8$ & $-7.11 \mathrm{e}-09$ \\
7 & $3.0 \mathrm{e}-8$ & $-5.10 \mathrm{e}-8$ & $-2.75 \mathrm{e}-06$ \\
9 & $-1.0 \mathrm{e}-7$ & $-4.50 \mathrm{e}-8$ & $-1.23 \mathrm{e}-07$ \\
11 & $7.0 \mathrm{e}-8$ & $-2.30 \mathrm{e}-8$ & $5.91 \mathrm{e}-09$ \\
\hline
\end{tabular}

TABLE III. PHASE ERRORS OF DIFFERENT WINDOW INTERPOLATION ALGORITHMS

\begin{tabular}{cccc}
\hline Harmonic & Blackman & Nuttall & Nuttall self-convolution window \\
\hline 1 & $-9.0 \mathrm{e}-8$ & $-4.0 \mathrm{e}-8$ & $8.12 \mathrm{e}-10$ \\
3 & $-1.0 \mathrm{e}-8$ & $-1.0 \mathrm{e}-8$ & $-1.12 \mathrm{e}-09$ \\
5 & $-1.3 \mathrm{e}-7$ & $-4.0 \mathrm{e}-8$ & $3.62 \mathrm{e}-10$ \\
7 & $-9.0 \mathrm{e}-8$ & $5.0 \mathrm{e}-8$ & $1.77 \mathrm{e}-05$ \\
9 & $-1.3 \mathrm{e}-7$ & $9.0 \mathrm{e}-8$ & $6.19 \mathrm{e}-07$ \\
11 & $8.0 \mathrm{e}-8$ & $6.0 \mathrm{e}-8$ & $-1.09 \mathrm{e}-09$ \\
\hline
\end{tabular}

TABLE IV. FREQUENCY ERRORS OF THE NUTTALL SELFCONVOLUTION WINDOW INTERPOLATION ALGORITHMS

\begin{tabular}{cc}
\hline Harmonic & Nuttall self-convolution window \\
\hline 1 & $-3.31 \mathrm{e}-12$ \\
3 & $4.58 \mathrm{e}-12$ \\
5 & $-1.48 \mathrm{e}-12$ \\
7 & $-7.22 \mathrm{e}-08$ \\
9 & $-2.52 \mathrm{e}-09$ \\
11 & $4.43 \mathrm{e}-12$ \\
\hline
\end{tabular}


The test result shows that the Nuttall self-convolution window could accurately measures the harmonic content of the line, and the detection result is higher accuracy. Especially for the frequency measurement, the error of the fundamental wave and the 3 or 5 times harmonic have reached e- 12 .

\section{B. Noise Impact Analysis}

In actual measurement, the signal often contains large amounts of background noise, resulting in lower resolution of the signal. In this paper, Gauss white noise with different signal-to-noise ratio is added to the signal, and the following data are obtained. As shown below, the Nuttall self-convolution window is still very accurate for noisy jamming signals.

TABLE V. AMPLITUDE ERRORS OF THE NUTTALL SELFCONVOLUTION WINDOW WITH DIFFERENT SIGNAL-TO-NOISE RATIO

\begin{tabular}{cccc}
\hline Harmonic & SNR=30db & SNR=50db & SNR=70db \\
\hline 1 & $7.17 \mathrm{e}-05$ & $1.92 \mathrm{e}-04$ & $-1.33 \mathrm{e}-05$ \\
3 & $-8.21 \mathrm{e}-04$ & $-1.18 \mathrm{e}-05$ & $2.31 \mathrm{e}-05$ \\
5 & $-4.77 \mathrm{e}-05$ & $1.42 \mathrm{e}-04$ & $-2.53 \mathrm{e}-05$ \\
7 & $4.55 \mathrm{e}-04$ & $-2.52 \mathrm{e}-04$ & $-3.95 \mathrm{e}-05$ \\
9 & $8.59 \mathrm{e}-04$ & $-6.62 \mathrm{e}-05$ & $1.99 \mathrm{e}-05$ \\
11 & $-7.34 \mathrm{e}-04$ & $1.65 \mathrm{e}-04$ & $-1.03 \mathrm{e}-05$ \\
\hline
\end{tabular}

TABLE VI. FREQUENCY ERRORS OF THE NUTTALL SELFCONVOLUTION WINDOW WITH DIFFERENT SIGNAL-TO-NOISE RATIO

\begin{tabular}{cccl}
\hline Harmonic & SNR=30db & SNR=50db & SNR=70db \\
\hline 1 & $2.90 \mathrm{e}-06$ & $-2.06 \mathrm{e}-06$ & $1.53 \mathrm{e}-07$ \\
3 & $-6.18 \mathrm{e}-05$ & $-2.54 \mathrm{e}-06$ & $8.63 \mathrm{e}-07$ \\
5 & $2.67 \mathrm{e}-05$ & $-5.64 \mathrm{e}-07$ & $9.29 \mathrm{e}-07$ \\
7 & $-5.12 \mathrm{e}-05$ & $7.43 \mathrm{e}-06$ & $-1.02 \mathrm{e}-07$ \\
9 & $1.10 \mathrm{e}-04$ & $2.19 \mathrm{e}-06$ & $-8.48 \mathrm{e}-07$ \\
11 & $8.69 \mathrm{e}-05$ & $-3.80 \mathrm{e}-06$ & $1.08 \mathrm{e}-06$ \\
\hline
\end{tabular}

\section{CONCLUSION}

In this paper, 4 order 5 Nuttall windows are used to construct Nuttall self-convolution window by $p$ order self convolution. The analysis shows that the sidelobe peak level and sidelobe attenuation rate of the Nuttall self-convolution window are proportional to the order of convolution. In the asynchronous sampling, the cosine combination window has better spectrum leakage suppression ability. Combined with the improved FFT algorithm, the high accuracy analysis of signal harmonics under non steady state can be achieved. Simulation results verify the correctness and effectiveness of the algorithm. Compared with the existing algorithms, the accuracy of the proposed algorithm is improved significantly, which is beneficial to embedded system applications. The simulation results showed that the proposed method have a higher accuracy of harmonic analysis and could improve the anti-noise performance.

\section{REFERENCES}

[1] Pang Hao, Li Dongxia, Zu Yunxiao, et a1. An improved algorithm for harmonic analysis of power system using FFT Technique [J].Proceedings of the CSEE, 2003, 23(6) : 50-54.
[2] ZENG Bo, TENG Zhaosheng. A Nuttall self-convolution window-based approach to weighted analysis on power system harmonic[J]. Power System Technology,2011, 35(8): 134-139.

[3] ZENG Bo, TENG Zhaosheng, WEN He, et al. Harmonic analysis based on Rife-Vincent window interpolated FFT[J]. Proceeding of the CSEE, 2009, 29(10): 115-120.

[4] ZENG Bo, TENG Zhao-sheng, GAO Yun-peng, et al. An accurate approach for power harmonic phasor calculation based on Rife-Vincent window[J]. Transactions of China Electrotechnical Society, 2009, 24(8): 154-159.

[5] LI Demin, HE Yigang. Harmonic analysis of power system based on Nuttall window four-spectrum-line interpolation FFT[J]. Power System protection and Control, 2016,44(3):64-71.

[6] ZENG Bo, TANG Qiu, QING Baiyuan, et al. Spectral analysis method based on improved FFT by Nuttall self-convolution window[J]. Transactions of China Electrotechnical Society, 2014, 29(7): 59-65.

[7] Yang Yuxiang,Zhang Fu,Qiao Yang, et al. Multi-frequency Synchronized Measurement Method of Bioimpedance Spectroscopy Based on Nuttall Self-convolution Windowed FFT Algorith[J].Journal of Electronics \& Information Technology, 2014,36(1): 250-254.

[8] NIU Shengsuo, LIANG Zhirui, ZHANG Jianhua, et al.An algorithm for electrical harmonic analysis based on triple-spectrum-1ine interpolation FFT[J]. Proceedings of the CSEE, 2012, 32(16): 130-136.

[9] HUANG Dongmei, GONG Renxi, JIAO Fengchang, et al.Power harmonic analysis based on Rife-Vincent window and triple-spectral-line interpolation[J]. Power System Protection and Control, 2014, 42(2): 2834. 